\title{
An Iterative Procedure for Computing the Maximal Root of a Positive Matrix
}

\author{
By T. L. Markham
}

I. Introduction. A. Kolmogoroff proved the following theorem [2].

Theorem A. Suppose $A$ is a positive matrix of order $n$. Then $A$ is similar to a positive generalized stochastic matrix with each row sum equal to the maximal positive characteristic root, $\lambda(A)$, of $A$.

The proof of Theorem A depends upon the fact that each positive matrix has a positive characteristic vector corresponding to the maximal positive characteristic root.

Professor A. T. Brauer developed a practical method in [1] for computing the maximal characteristic root of a positive matrix to any desired degree of accuracy. In particular, he proved

Theorem B. Let $A$ be a positive square matrix of order $n$, and let $R(r)$ denote the largest (smallest) row sum of $A$. If $\epsilon>0$, there exists a matrix, $F(\epsilon)$, which is similar to $A$ such that $R^{*}-r^{*}<\epsilon$, where $R^{*}\left(r^{*}\right)$ denotes the largest (smallest) row sum of $F(\epsilon)$.

It is known that $r \leqq \lambda(A) \leqq R$ [1, Theorem 13 , p. 21], and the inequality is strict unless $R=r$. Thus it follows immediately from Theorem $\mathrm{B}$ that $\lambda(A)$ can be computed to any desired degree of accuracy.

We shall offer a new method for computing the maximal root of a positive matrix.

II. An Iterative Procedure for Computing the Maximal Root. We shall use the following notation. Suppose $A$ is a positive matrix of order $n$. Let $R_{i}$ denote the $i$ th row sum of $A, R=\max _{i}\left\{R_{i}\right\}$, and $r=\min _{i}\left\{R_{i}\right\}$.

Theorem 1. Suppose $A$ is a positive matrix of order $n$, with $R>r$. Let $S=$ $\operatorname{diag}\left(R_{1}, \cdots, R_{n}\right)$. Then $S^{-1} A S$ is a positive matrix with each row sum in the interval $(r, R)$.

Proof. Assume, without loss of generality, that $R=R_{1} \geqq R_{2} \geqq \cdots \geqq R_{n}=r$. Then $S^{-1} A S=\left(a_{i j} R_{j} / R_{i}\right)$ is a positive matrix. Moreover,

$$
\frac{\sum_{j=1}^{n} a_{i j} R_{j}}{R_{i}}<\frac{\sum_{j=1}^{n} a_{i j} R_{1}}{R_{i}}=R,
$$

and

$$
\frac{\sum_{j=1}^{n} a_{i j}}{R_{i}} R_{j}>\frac{\sum_{j=1}^{n} a_{i j} R_{n}}{R_{i}}=r, \text { for } i=1,2, \cdots, n \text {. }
$$

Hence each row sum of $S^{-1} A S$ lies in the interval $(r, R)$.

If we denote $B_{1}=S^{-1} A S$, then we can transform $B_{1}$ by a similarity transformation, obtaining $B_{2}$, such that each row sum of $B_{2}$ lies in the interval determined

Received March 27, 1968. 
by the largest and smallest row sums of $B_{1}$. We continue in this manner and obtain a sequence $B_{1}, B_{2}, \cdots, B_{n}, \cdots$ such that the difference of the largest row sum and the smallest row sum of $B_{i}$ is decreasing at each step. Theorem 2 assures us that we do obtain convergence to a positive generalized stochastic matrix.

Theorem 2. Suppose $A$ is a positive matrix of order $n$, and let $B_{1}, B_{2}, \cdots$, $B_{n}, \cdots$ denote the sequence of matrices obtained from $A$ by the procedure outlined above. Assume $a_{k 1} / R_{k}=\min _{i}\left\{a_{i 1} / R_{i}\right\}$. Then

max row sum of $B_{n}-\min$ row sum of $B_{n} \leqq\left(1-a_{k 1} / R_{k}\right)^{n}(R-r)$.

I'roof. Note the following inequalities:

$$
\text { ith row sum of } \begin{aligned}
B_{1} & =\sum_{j=1}^{n} a_{i j} \frac{R_{j}}{R_{i}} \leqq \sum_{j=1}^{n-1} \frac{a_{i j} R}{R_{i}}+\frac{a_{i n} r}{R_{i}}=\sum_{j=1}^{n-1} \frac{a_{i j}(R-r)}{R_{i}}+r \\
& =\left(1-\frac{a_{i n}}{R_{i}}\right)(R-r)+r=\left(1-\frac{a_{i n}}{R_{i}}\right) R+\frac{a_{i n}}{R_{i}} r .
\end{aligned}
$$

Assume $\min _{i}\left\{a_{i n} / R_{i}\right\}=a_{p n} / R_{p}$. Then

$$
i \text { th row sum of } B_{1} \leqq\left(1-a_{p n} / R_{p}\right) R+\left(a_{p n} / R_{p}\right) r \text { for each } i .
$$

Similarly, we have

$$
i \text { th row sum of } B_{1} \geqq\left(1-a_{k 1} / R_{k}\right) r+\left(a_{k 1} / R_{k}\right) R \text { for each } i,
$$
where $a_{k 1} / R_{k}=\min _{i}\left\{a_{i 1} / R_{i}\right\}$.

Using (1) and (2), it follows that

max row sum of $B_{1}-\min$ row sum of $B_{1}$

$$
\begin{aligned}
& \leqq\left(1-a_{p n} / R_{p}\right) R+\left(a_{p n} / R_{p}\right) r-\left[\left(1-a_{k 1} / R_{k}\right) r+\left(a_{k 1} / R_{k}\right) R\right] \\
& =\left(1-\left[a_{p n} / R_{p}+a_{k 1} / R_{k}\right]\right)(R-r)<\left(1-a_{k 1} / R_{k}\right)(R-r) .
\end{aligned}
$$

Hence the result holds when $n=1$; i.e.

$$
\text { max row sum of } B_{1}-\text { min row sum of } B_{1}<\left(1-a_{k 1} / R_{k}\right)(R-r) \text {. }
$$

In the same manner, it is true that

$$
\text { max row sum of } B_{2}-\text { min row sum of } B_{2}
$$

$$
\leqq\left(1-\min _{i}\left\{\frac{a_{i 1} R_{1}}{\sum_{j=1}^{n} a_{i} ; R_{j}}\right\}\right)\left(\text { max row sum of } B_{1}-\text { min row sum of } B_{1}\right) \text {. }
$$

But

$$
\frac{a_{i 1} R_{1}}{\sum_{j=1}^{n} a_{i j} R_{j}} \geqq \frac{a_{i 1} R_{1}}{\sum_{j=1}^{n} a_{i j} R_{1}} \geqq \frac{a_{k 1}}{R_{k}} \text { for each } i
$$

Hence

$$
\min _{i}\left\{\frac{a_{i 1} R_{1}}{\sum_{j=1}^{n} a_{i j} R_{j}}\right\} \geqq \frac{a_{k 1}}{R_{k}} .
$$

Finally, with (4) and (5), we have

$$
\text { max row sum of } B_{2}-\min \text { row sum of } B_{2} \leqq\left(1-a_{k 1} / R_{k}\right)^{2}(R-r) \text {. }
$$


The remainder of the proof is an easy induction.

Theorem 1 yields a simple method for the computation of the maximal positive root of a positive matrix.

III. An Example. The following computations were performed at the University of North Carolina at Charlotte Computer Center, and the author gratefully acknowledges the use of an algorithm prepared by Professors D. Nixon and L. Davis.

Let

$$
\begin{aligned}
& A=\left(\begin{array}{rrrr}
4 & 7 & 25 & 2 \\
1 & 12 & 5 & 18 \\
3 & 6 & 15 & 2 \\
5 & 2 & 2 & 1
\end{array}\right) \\
& \text { min row sum max row sum } \\
& \text { A : } \quad 10 \quad 38 \\
& B_{1}: \quad 21.6666 \quad 32.4 \\
& B_{2}: \quad 25.5974 \quad 26.9567 \\
& B_{3}: \quad 26.2425 \quad 26.6185 \\
& B_{4}: \quad 26.3759 \quad 26.4030 \\
& B_{5}: \quad 26.3807 \quad 26.3931 \\
& B_{6}: \quad 26.3831 \quad 26.3873 \\
& B_{7}: \quad 26.3845 \quad 26.3853 \\
& B_{8}: \quad 26.3849 \quad 26.3850
\end{aligned}
$$

All numbers are truncated at four decimal places, and the computations were performed in double-precision arithmetic.

The University of North Carolina

Mathematics Department

Charlotte, North Carolina 28205

The University of South Carolina

Mathematics Department

Columbia, South Carolina 29208

1. Alfred Brauer, "On the characteristic roots of non-negative matrices," Recent Advances in Matrix Theory, (Proc. Advanced Seminar, Math. Res. Center, U. S. Army), Univ. of Wisconsin Press, Madison, Wis., 1964, pp. 3-38. MR 29 \#5835.

2. N. DMITRIEv \& E. DYNKIN, "On characteristic roots of stochastic matrices," Bull. Acad. Sci. URSS Sér. Math., v. 10, 1946, pp. 167-184. MR 8, 129. 